\title{
Singular Filters for the Radon Backprojection
}

\section{Journal Article}

\section{Author(s):}

Hungerbühler, N.

Publication date:

1999

Permanent link:

https://doi.org/10.3929/ethz-b-000422767

\section{Rights / license:}

In Copyright - Non-Commercial Use Permitted

\section{Originally published in:}

Journal of Applied Analysis 5(1), https://doi.org/10.1515/JAA.1999.17 


\title{
SINGULAR FILTERS FOR THE RADON BACKPROJECTION
}

\author{
N. HUNGERBÜHLER
}

Received April 22, 1997 and, in revised form, May 20, 1998

\begin{abstract}
Numerous methods for the inversion of the Radon transformation which is the basis of computerized tomography are known: Fourier inversion, filtered backprojection or Kaczmarz's method. In the filtered backprojection method the choice of the filter function is crucial for the quality of the pictures. Here we deal with singular filter functions which have some advantages in comparison with conventional filters. An adapted method allows to handle the numerical problems caused by the singularities.
\end{abstract}

\section{Introduction}

The method of computerized tomography is used in many fields: medicine, geology, astrophysics etc. All those applications are based on the following model: An X-ray of initial intensity $I_{0}$ has after passing through a material of optical density $f$ and thickness $\mu$ a reduced intensity $I(\mu)=$ $I_{0} \exp (-f \mu)$. If the material is non-homogeneous, i.e. if $f$ is a function of $x$, and if we neglect optical refraction, then the X-ray follows a straight line

1991 Mathematics Subject Classification. 65R10, 65C20, 65B05.

Key words and phrases. Radon transformation, integral transformation, backprojection method, singular integral kernels, filter function, numerical image reconstruction.

ISSN 1425-6908 (c) Heldermann Verlag. 
$L$ and its final intensity becomes

$$
I(L)=I_{0} \exp \left(-\int_{L} f(x) d \tau\right)
$$

where $d \tau$ denotes the line element on $L . \quad \int_{L} f(x) d \tau$ is called the Radon transform of $f$ as a function of $L$. Thus by measuring one can find the value of $\int_{L} f(x) d \tau$ for every line $L$ in a given plane $\Pi$. The problem is then to reconstruct the function $f$ from those data. Radon found the solution in 1917. For the reader who is not familiar with the theory we include a brief introduction in Section 1.1 which follows [8, Chapter II].

A glance at Radon's original formula explains why the problem is eighty years later still a matter of discussion. In fact, Radon found the following explicit representation of the solution:

$$
f(x)=-\frac{1}{\pi} \int_{0}^{\infty} \frac{d F_{x}(q)}{q}
$$

with

$$
F_{x}(q)=\frac{1}{2 \pi} \int_{S^{1}} R f(\theta, x \cdot \theta+q) d \theta
$$

where $R f$ denotes the Radon transform of $f$ (see Section 1.1 for the definition). Unfortunately, this formula exhibits a bad numerical behavior (even if the Radon transform $R f$ would be known on its full domain of definition) and is therefore of no practical use. Moreover, in practice, $R f$ is only given by a discrete number of measurements, and it can be shown that the set of $f$ leading to the same finite set of data is very large (see Section 1.2). A direct implementation of Radon's formula does not lead to a reasonable reconstruction. Therefore, many alternative methods were invented which allow to control the properties of the reconstructed function in terms of the original function (see Section 1.3). The aim of this paper is to show, that the use of singular filters in the backprojection method has some advantages compared to classical filter functions.

\subsection{Basic definitions and properties of the Radon transformation.}

Definition 1. The Radon transformation is given by

$$
\begin{aligned}
R: \mathcal{S}\left(\mathbb{R}^{2}\right) & \rightarrow \mathcal{S}(Z) \\
f & \mapsto R f(\theta, s):=\int_{\theta \cdot x=s} f(x) d \tau
\end{aligned}
$$

where $\mathcal{S}\left(\mathbb{R}^{2}\right)$ and $\mathcal{S}(Z)$ denote the Schwartz-space on $\mathbb{R}^{2}$ and on the cylinder $Z=S^{1} \times \mathbb{R}$ respectively. We use the notation $R_{\theta} f(s)$ if we consider the vector $\theta \in S^{1}$ as fixed. 
Remark. Of course this definition may be extended to larger classes of functions.

An easy inversion formula is based on the Fourier transformation:

Theorem 1. For $f \in \mathcal{S}\left(\mathbb{R}^{2}\right)$ there holds $\widehat{R_{\theta} f}(\sigma)=\sqrt{2 \pi} \widehat{f}(\sigma \theta)$.

Proof. By definition we have

$$
\begin{aligned}
\widehat{R_{\theta} f}(\sigma) & =\frac{1}{\sqrt{2 \pi}} \int_{\mathbb{R}} \exp (-i s \sigma) R_{\theta} f(s) d s= \\
& =\frac{1}{\sqrt{2 \pi}} \int_{\mathbb{R}} \exp (-i s \sigma) \int_{\theta^{\perp}} f(s \theta+y) d y d s .
\end{aligned}
$$

Substituting $x:=s \theta+y$, i.e. $s=\theta \cdot x$ and $d x=d y d s$, we get

$$
\widehat{R_{\theta} f}(\sigma)=\frac{1}{\sqrt{2 \pi}} \int_{\mathbb{R}^{2}} \exp (-i \sigma \theta \cdot x) f(x) d x=\sqrt{2 \pi} \widehat{f}(\sigma \theta) .
$$

This inversion formula is the basis in the Fourier-reconstruction method. The backprojection method which we want to use here needs the following definition:

Definition 2. The dual Radon transformation is given by

$$
\begin{aligned}
R^{\#}: \mathcal{S}(Z) & \rightarrow \mathcal{S}\left(\mathbb{R}^{2}\right) \\
g & \mapsto R^{\#} g(x):=\int_{S^{1}} g(\theta, \theta \cdot x) d \theta
\end{aligned}
$$

The filtered backprojection method is based upon the following theorem connecting Radon and dual Radon transformation by the convolution.

Theorem 2. For $f \in \mathcal{S}\left(\mathbb{R}^{2}\right)$ and $W \in \mathcal{S}(Z)$ there holds

$$
f *\left(R^{\#} W\right)=R^{\#}(R f * W) .
$$

Proof.

$$
\begin{aligned}
f *\left(R^{\#} W\right)(x) & =\int_{\mathbb{R}^{2}} R^{\#} W(x-y) f(y) d y= \\
& =\int_{\mathbb{R}^{2}} \int_{S^{1}} W(\theta, \theta \cdot(x-y)) d \theta f(y) d y= \\
& =\int_{S^{1}} \int_{\mathbb{R}^{2}} W(\theta, \theta \cdot(x-y)) f(y) d y d \theta
\end{aligned}
$$


Substituting $y:=s \theta+z, z \in \theta^{\perp}$, we obtain

$$
\begin{aligned}
f *\left(R^{\#} W\right)(x) & =\int_{S^{1}} \int_{\mathbb{R}} \int_{\theta^{\perp}} W(\theta, \theta \cdot x-s) f(s \theta+z) d z d s d \theta= \\
& =\int_{S^{1}} \int_{\mathbb{R}} W(\theta, \theta \cdot x-s) R f(\theta, s) d s d \theta= \\
& =\int_{S^{1}}(W * R f)(\theta, \theta \cdot x) d \theta .
\end{aligned}
$$

If we choose in the above theorem a function $W$ such that $w=R^{\#} W$ is in some sense an approximation of the delta distribution then we get

$$
f \approx f * w=R^{\#}(R f * W) .
$$

This is the heart of the filtered backprojection method. The functions $w$ respectively $W$ are called filter. The filtered backprojection method consists in an implementation of the right hand side of (1).

For other inversion formulas, such as Radon's original formula, we refer to [8, Section II.2].

Below it will be necessary to invert $R^{\#}$. The following theorem enables us to do this.

Theorem 3. For $g \in \mathcal{S}(Z)$ there holds

$$
\widehat{R^{\#}} g(\xi)=\frac{\sqrt{2 \pi}}{|\xi|}\left(\widehat{g}\left(\frac{\xi}{|\xi|},|\xi|\right)+\widehat{g}\left(-\frac{\xi}{|\xi|},-|\xi|\right)\right) \text {. }
$$

Proof. (i) For $f \in \mathcal{S}\left(\mathbb{R}^{2}\right)$ we have

$$
\begin{aligned}
\int_{\mathbb{R}} R_{\theta} f(s) g(\theta, s) d s & =\int_{\mathbb{R}} \int_{\theta^{\perp}} f(\theta s+y) g(\theta, s) d y d s= \\
& =\int_{\mathbb{R}^{2}} f(x) g(\theta, \theta \cdot x) d x .
\end{aligned}
$$

Integration over $S^{1}$ yields

$$
\int_{S^{1}} \int_{\mathbb{R}} R f(\theta, s) g(\theta, s) d s d \theta=\int_{\mathbb{R}^{2}} f(x) R^{\#} g(x) d x .
$$

(ii) Let $\widehat{w} \in \mathcal{S}\left(\mathbb{R}^{2}\right)$. Then (i) gives

$$
\begin{aligned}
\int_{\mathbb{R}^{2}} \widehat{w}(x) R^{\#} g(x) d x & =\int_{S^{1}} \int_{\mathbb{R}} g(\theta, s) R \widehat{w}(\theta, s) d s d \theta= \\
& =\int_{S^{1}} \int_{\mathbb{R}} \widehat{g}(\theta, \sigma)(R \widehat{w})^{\mathrm{v}}(\theta, \sigma) d \sigma d \theta .
\end{aligned}
$$


In the last line we used Parseval's identity (the Fourier transformation for functions in $\mathcal{S}(Z)$ only acts on the second argument). From Theorem 1 we infer that $(R \widehat{w})^{\mathrm{v}}(\theta, \sigma)=\sqrt{2 \pi} w(\theta \sigma)$. Hence

$$
\begin{aligned}
\int_{\mathbb{R}^{2}} \widehat{w}(x) R^{\#} g(x) d x & =\sqrt{2 \pi} \int_{S^{1}} \int_{\mathbb{R}} \widehat{g}(\theta, s) w(\theta \sigma) d \sigma d \theta= \\
& =\sqrt{2 \pi} \int_{\mathbb{R}^{2}}\left(\widehat{g}\left(\frac{\xi}{|\xi|},|\xi|\right)+\widehat{g}\left(-\frac{\xi}{|\xi|},-|\xi|\right)\right) \frac{w(\xi)}{|\xi|} d \xi .
\end{aligned}
$$

Here we used $\xi=\theta \sigma$ once on $\sigma<0$, once on $\sigma>0$. From Parseval's identity the assertion follows since $w$ was arbitrary.

1.2. Uniqueness. According to Theorem 1 the Radon transformation is bijective. But how is the situation if $R f$ is known only on a subset of its domain of definition as it is typically the case in practice? The following theorem shows that we should not hope for too much. (For the proof we refer to [8, Theorem II.3.7].)

Theorem 4. Let $\theta_{1}, \ldots, \theta_{p} \in S^{1}$ be distinct directions, $K \subset \mathbb{R}^{2}$ a compact set and $f \in C_{0}^{\infty}(K)$. Then for every compact set $K_{0} \subset \stackrel{\circ}{K}$ in the interior of $K$ there exists $f_{0} \in C_{0}^{\infty}(K)$ having the property that $f_{0} \equiv f$ on the set $K_{0}$ and $R_{\theta_{i}} f_{0}=0$ for $1 \leq i \leq p$.

1.3. Resolution. In Section 1.2 we have seen that we cannot reconstruct $f$ from its Radon transform if $R f$ is only known on a finite set of directions $\theta_{i}$. Now we ask for the "size of details" that can be reconstructed depending on the choice of the $\theta_{i}$.

Definition 3. A function $f: \mathbb{R}^{n} \rightarrow \mathbb{R}$ is called $b$-band-limited if its Fourier transform vanishes outside a ball of radius $b$.

Example 1. The function

$$
\operatorname{sinc}(x):= \begin{cases}\frac{\sin (x)}{x} & \text { if } x \neq 0 \\ 1 & \text { if } x=0\end{cases}
$$

is 1-band limited since $\widehat{\operatorname{sinc}}(\xi)=\sqrt{\pi / 2} \chi_{[-1,1]}(\xi)$ (where $\chi_{A}$ denotes the characteristic function of the set $A)$. If we consider $\operatorname{sinc}(x)=\prod_{i=1}^{n} \operatorname{sinc}\left(x_{i}\right)$ on $\mathbb{R}^{n}$ then $\operatorname{sinc}_{b}(x)=\operatorname{sinc}(b x)$ is $\sqrt{n} b$-band-limited since $\widehat{\operatorname{sinc}} b=(\pi / 2)^{n / 2} \times$ $b^{-n} \chi_{[-b, b]^{n}}$. 
We say that $\operatorname{sinc}_{b}$ (interpreted as density function of a picture) represents a detail of size $2 \pi / b$. Hence a $b$-band-limited function does not contain details smaller than $2 \pi / b$.

A picture typically has finite extension, i.e. its density function $f$ has compact support and hence its Fourier transform is analytic and cannot vanish outside a ball unless it is identically zero. Thus $f$ cannot be $b$-bandlimited in the strong sense. So we say a function $f$ is essentially b-bandlimited if $\widehat{f}$ is small (in a sense that has to be made precise) outside a ball of radius $b$. Essentially $b$-band-limited functions are interpreted analogously as $b$-band-limited functions.

Theorem 5. Let $f \in C_{0}^{\infty}(\Omega)$ for an open domain $\Omega$ in $\mathbb{R}^{2}$ and $g(\phi, s)=$ $R f(\theta, s)$ for $\theta=\left(\begin{array}{c}\cos (\phi) \\ \sin (\phi)\end{array}\right)$. For $0<\vartheta<1$ and $b \geq 1$ we define

$$
K:=\left\{(k, \sigma):|\sigma|<b,|k|<\max \left(\frac{|\sigma|}{\vartheta}, b\left(\frac{1}{\vartheta}-1\right)\right)\right\} \subset \mathbb{R}^{2} .
$$

Let $W \in G L_{2}(\mathbb{R})$ having the property that the sets $K+2 \pi W^{-T} n$ are disjoint for $n \in \mathbb{Z}^{2}$. Then there holds

$$
\|R f\|_{L^{\infty}(\Omega)} \leq \eta(\vartheta, b)\|f\|_{L^{1}(\Omega)}+\frac{8}{\pi \vartheta} \int_{|\xi|>b}|\widehat{f}(\xi)| d \xi
$$

provided $g(W n)=0$ for $n \in \mathbb{Z}^{2}$.

For a proof we refer to [8, Theorem III.3.1].

Theorem 5 is interpreted as follows: If $f$ is essentially $b$-band-limited in the sense that $\int_{|\xi|>b}|\widehat{f}(\xi)| d \xi$ is small then $f$ may be reconstructed from the values of $R f$ on the grid $W n, n \in \mathbb{Z}^{2}$, with the above error estimate provided the sets $K+2 \pi W^{-T} n$ are disjoint for $n \in \mathbb{Z}^{2}$.

The standard sampling geometry is

$$
W=\left(\begin{array}{cc}
\pi / p & 0 \\
0 & 1 / q
\end{array}\right)
$$

i.e. $R f$ is measured for the $p$ directions $\theta_{i}=i(\pi / p), 1 \leq i \leq p$, and for every direction $\theta_{i}$ at the points $n / q, n \in \mathbb{Z}$. The points $K+2 \pi W^{-T} n$ are disjoint if the so called sampling conditions

$$
b \leq p \vartheta \text { and } b \leq \pi q
$$

hold. They are also valid for the reconstruction of $f * w$ since there holds

$$
|R(f * w)(\theta, s)| \leq\|R f\|_{L^{\infty}(\Omega)}\left|\int_{\mathbb{R}} R_{\theta} w(s-t) d t\right| \leq\|R f\|_{L^{\infty}(\Omega)}
$$

if $\|w\|_{L^{1}}=1$. 


\section{Singular filters}

Usually, a filter is chosen based on properties of $W$. Here we will choose $w$ first as the characteristic function of a pixel for several pixel shapes.

A filter function which is very often used in practice is $w_{b}$ defined by

$$
\widehat{w}_{b}(\xi)=\frac{1}{2 \pi} \widehat{\Psi}\left(\frac{|\xi|}{b}\right)
$$

for a parameter $\varepsilon \in[0,1]$ and

$$
\widehat{\Psi}(\sigma)= \begin{cases}1-\varepsilon \sigma & \text { for } \sigma \leq 1 \\ 0 & \text { else. }\end{cases}
$$

This is essentially the filter introduced by Ramachandran and Lakshminarayanan which is of the same type as the famous filter of Shepp and Logan (see e.g. [9], [7] or [2]). Recall that for the dual filter $W_{b}$ with $R^{\#} W_{b}=w_{b}$ we get the formula of the filtered backprojection $f(x) \approx$ $f * w_{b}(x)=R^{\#}(R f * W)(x)$ as discussed in Theorem 2. What are the disadvantages of this type of filters:

(I) The resulting function $w_{b}$ is $b$-band-limited. Since $w_{b}$ is analytic, it has non-compact support. This means that the value $f(x) \approx f * w_{b}(x)$ (which is constructed by the filtered backprojection method) involves also values of $f$ which are far away from $x$. In other words, if we change $f$ at some point $x$ this will influence all other values $f * w_{b}(y)$.

(II) Since the function $W_{b}$ is quite regular, the classical trapezoidal rule is usually applied in the calculation of the convolution $R f * W_{b}$. However if functions with large bandwidth have to be reconstructed, i.e. if $b$ has to be chosen large, $W_{b}$ has large second derivatives and (according to the theory of the trapezoidal rule) the error term is large.

Those two main errors cannot be made small at the same time, they are antagonistic.

So let us try a filter function

$$
w_{A}=\frac{1}{|A|} \chi_{A}
$$

where $A$ is just one pixel on the screen, i.e. a circle, a square or a hexagon (centered at the origin). Then $f * w_{A}(x)$ is exactly the mean value of $f$ over the pixel $A_{x}$ centered at the point $x$. This strategy has no longer the disadvantage (I). Unfortunately the dual function $W_{A}$ will be singular in general and the calculation of the convolution $R f * W_{A}$ in $R^{\#}\left(R f * W_{A}\right)$ becomes a numerical problem. This is clearly the reason why this kind of filter has not been discussed in the literature yet, although variants may be found in [10], [9] or [7]. However if we use a modification of the classical 
trapezoidal rule (see next section) we overcome this difficulty. The resulting algorithm will not be slower than the classical filtered backprojection method. Let us first calculate the filters $W_{A}$ for three different shapes of pixels $A$.

2.1. A hexagon. Let us consider the weight function

$$
w_{\lambda}=\frac{1}{\left|H_{\lambda}\right|} \chi_{H_{\lambda}}
$$

where $H_{\lambda}$ denotes the regular hexagon with side length $\lambda$ centered at the origin and oriented such that one side is parallel to the $x$-axes. The area of this hexagon is $\left|H_{\lambda}\right|=(3 \sqrt{3} / 2) \lambda^{2}$. We have to find $W_{\lambda}$ such that $R^{\#} W_{\lambda}=$ $w_{\lambda}$. We calculate the case $\lambda=1$. Then from $R^{\#} W_{1}=w_{1}$ the general case follows by scaling

$$
W_{\lambda}(\theta, s)=\frac{1}{\lambda^{2}} W_{1}\left(\theta, \frac{s}{\lambda}\right) .
$$

We are free to assume that $W_{1}$ is even, i.e. $W_{1}(-\theta,-s)=W_{1}(\theta, s)$. Thus Theorem 3 gives

$$
\widehat{W}_{1}(\theta, \sigma)=\frac{1}{2 \sqrt{2 \pi}}|\sigma| \widehat{w}_{1}(\theta \sigma) .
$$

Remark. Note that this is only a formal relation since the hypothesis that $w_{1} \in \mathcal{S}\left(\mathbb{R}^{2}\right)$ is clearly not fulfilled. We can take (9) as a definition of $W_{1}$ and show after formal calculation of $W_{1}$ that in fact $R^{\#} W_{1}=w_{1}$ a.e. and that $f * w_{\lambda}=R^{\#}\left(R f * W_{\lambda}\right)$.

Let the vectors $a_{k}$ denote the edges of $H_{1}$

$$
a_{k}=\left(\begin{array}{l}
\operatorname{Re}(\exp (2 i k \pi / 3)) \\
\operatorname{Im}(\exp (2 i k \pi / 3))
\end{array}\right) \text {. }
$$

Then a quite extensive calculation leads to

$$
\widehat{\chi}_{H_{1}}(\eta)=-\frac{\left|H_{1}\right|}{3 \pi} \frac{\eta \cdot a_{1} \cos \left(\eta \cdot a_{1}\right)+\eta \cdot a_{2} \cos \left(\eta \cdot a_{2}\right)+\eta \cdot a_{3} \cos \left(\eta \cdot a_{3}\right)}{\left(\eta \cdot a_{1}\right)\left(\eta \cdot a_{2}\right)\left(\eta \cdot a_{3}\right)}
$$

provided $\theta \cdot a_{k} \neq 0$. Using expression (10) in (7) and (8) we find (for $\left.\theta \cdot a_{k} \neq 0\right)$

$$
W_{\lambda}(\theta, s)=\frac{1}{12 \lambda^{2} \pi^{2}} \frac{\sum_{i=1}^{3} \theta \cdot a_{i} \log \left|\left(\theta \cdot a_{i}\right)^{2}-\frac{s^{2}}{\lambda^{2}}\right|}{\prod_{i=1}^{3} \theta \cdot a_{i}} .
$$


For $\theta \cdot a_{1}=0$ we find

$$
\widehat{\chi}_{H_{1}}(\eta)=\frac{\left|H_{1}\right|}{3 \pi\left(\eta \cdot a_{2}\right)\left(\eta \cdot a_{3}\right)}\left(\cos \left(\eta \cdot a_{2}\right)-\eta \cdot a_{2} \sin \left(\eta \cdot a_{2}\right)-1\right) .
$$

Now the function

$$
\widehat{W}_{1}(\theta, \sigma)=\frac{1}{\sqrt{2 \pi} 6 \pi|\sigma| \beta^{2}}(\cos (\sigma \beta)-\sigma \beta \sin (\sigma \beta)-1)
$$

(with $\beta:=\theta \cdot a_{2}=-\theta \cdot a_{3}$ ) does not have a Fourier inverse. However we can extend the function $W_{1}(\theta, s)$ continuously in the points $(\theta, s)$ where $\theta \cdot a_{1}=0$ :

$$
\lim _{\theta \cdot a_{1} \rightarrow 0} W_{1}(\theta, s)=\frac{1}{12 \pi^{2}}\left(\frac{2}{\alpha^{2}-s^{2}}-\frac{1}{\alpha^{2}} \log \left|\frac{s^{2}}{\alpha^{2}-s^{2}}\right|\right)
$$

with $\alpha=\lim _{\theta \cdot a_{1} \rightarrow 0} \beta=\sqrt{3} / 2$. The cases $\eta \cdot a_{2}=0$ and $\eta \cdot a_{3}=0$ are analogous. By direct calculation we can check that in fact $R^{\#} W_{1}(x)=$ $\left(\widehat{w}_{1}\right)^{\mathrm{v}}(x)=w_{1}(x)$ a.e. and that $f * w_{\lambda}=R^{\#}\left(R f * W_{\lambda}\right)$.

2.2. A square. Repeating the above calculations for the square $Q_{\lambda}$ with side length $2 \lambda$, centered at the origin with sides parallel to the axes we find that for $w_{\lambda}=\left(1 /\left|Q_{\lambda}\right|\right) \chi_{Q_{\lambda}}$ the dual filter has the form

$$
W_{\lambda}(\theta, s)=\frac{1}{4 \pi^{2} \lambda^{2}((a+b) \cdot \theta)((a-b) \cdot \theta)} \log \left|\frac{(a \cdot \theta)^{2}-\left(s^{2} / \lambda^{2}\right)}{(b \cdot \theta)^{2}-\left(s^{2} / \lambda^{2}\right)}\right|
$$

with $a=\left(\begin{array}{l}1 \\ 1\end{array}\right)$ and $b=\left(\begin{array}{c}1 \\ -1\end{array}\right)$ denoting the edges of $Q_{1}$. Again one can extend continuously this function if $\theta$ is perpendicular to one of the sides of the square.

2.3. A circle. Here we consider $K_{\lambda}$ the circle with radius $\lambda$ centered at the origin and $w_{\lambda}=\left(1 /\left|K_{\lambda}\right|\right) \chi_{K_{\lambda}}$. We know that

$$
\widehat{\chi}_{K_{1}}(\eta)=\frac{1}{|\eta|} J_{1}(|\eta|)
$$

where $J_{1}$ denotes the Bessel function of the first kind with index 1. Thus we obtain from (9) that

$$
\widehat{W}_{1}(\theta, \sigma)=\frac{1}{(2 \pi)^{3 / 2}} J_{1}(|\sigma|) .
$$


Fourier inversion leads to

$$
\begin{aligned}
W_{1}(\theta, s) & =\frac{1}{2 \pi^{2}} \int_{0}^{\infty} \cos (s \sigma) J_{1}(\sigma) d \sigma= \\
& = \begin{cases}\frac{1}{2 \pi^{2}}\left(1-\frac{|s|}{\sqrt{s^{2}-1}}\right) & \text { if }|s|>1 \\
\frac{1}{2 \pi^{2}} & \text { else. }\end{cases}
\end{aligned}
$$

This filter has the advantage that it does not depend on the angle $\theta$.

Now of course we have a problem to calculate numerically $R^{\#}\left(R f * W_{\lambda}\right)$ since in all of the considered cases the filter functions $W_{\lambda}$ have severe singularities. In the next section we present a method to handle this problem.

\section{A numerical method for product integration}

In this section we present a method to find a numerical approximation of the integral

$$
\int_{\mathbb{R}} f(x) \psi(x) d x
$$

where $f$ is supposed to be a "nice" function with compact support whereas $\psi$ may be discontinuous, unbounded or oscillating with high frequency. Then the standard integration algorithms as Simpson, Romberg or adaptive quadrature method do not converge in an appropriate way. In case of an unbounded $\psi$ the step-length at least in a neighborhood of the singular points of $\psi$ has to be very small-although $f$ could be nice all over the interval of integration. We have the further restriction that an algorithm which only evaluates $f$ at equidistant points is required.

The trapezoidal rule we present here can be found in [5] or [6].

Let $f \in C^{2}([a, b])$ and $\theta^{\prime \prime}=\psi \in L^{1}([a, b])$ and let $\Delta_{h}^{2}$ denote the central second difference quotient

$$
\Delta_{h}^{2} \theta(x)=\frac{\theta(x-h)-2 \theta(x)+\theta(x+h)}{h^{2}} .
$$

Then we have

$$
\int_{A}^{B} f(x) \psi(x) d x=h \sum_{i=1}^{n-1} f\left(a_{i}\right) \Delta_{h}^{2} \theta\left(a_{i}\right)+T[f]+R[f] .
$$

with boundary terms $T[f]$ (which vanish for $f \in C_{0}^{2}([A, B])$ ),

$$
\begin{aligned}
T[f]=f(B) \theta^{\prime}(B) & -f(A) \theta^{\prime}(A) \\
& +\frac{1}{h}\left(f(A)\left(\theta\left(a_{1}\right)-\theta(A)\right)+f(B)\left(\theta\left(a_{n-1}\right)-\theta(B)\right)\right)
\end{aligned}
$$


and error term $R[f]$,

$$
|R[f]| \leq \frac{h^{2}}{8} M_{A}^{B}\left(f^{\prime \prime}\right) \int_{A}^{B}|\psi(x)| d x .
$$

Definition. We call

$$
J[f]:=h \sum_{i=1}^{n-1} f\left(a_{i}\right) \Delta_{h}^{2} \theta\left(a_{i}\right)+T[f]
$$

trapezoidal rule. We shall use the notation $J_{h}[f]$ when we want to emphasize the dependence on $h$.

Interpretation. When we integrate $\int_{A}^{B} f(x) \theta^{\prime \prime}(x) d x$ for $f \in C_{0}^{2}([A, B])$ we have to replace the second differential quotient $\theta^{\prime \prime}$ by the second difference quotient $\Delta_{h}^{2} \theta$ and proceed as in the ordinary trapezoidal rule with mesh constant $h$ (when $f$ does not vanish at the endpoints of the interval a boundary term $T[f]$ has to be added). The error order is $O\left(h^{2}\right)$ as for the classical trapezoidal rule.

From [6] we infer the following extrapolation scheme for the trapezoidal formula (15).

Theorem 6. Let $\psi$ have finitely many algebraic singularities on an integration interval $I$ of length $l$. Let $f$ be smooth and $T_{1, n}=J_{l / 2^{n}}[f], n \in \mathbb{N}$, the numerical approximations for $\int_{I} f(x) \psi(x) d x$ obtained by the trapezoidal rule (15) applied with step length $h=l / 2^{n}$. If we denote by $T_{m+1, n}$, $m=1,2, \ldots$, the Aitken transformation of the sequence $T_{m, n}$, i.e.

$$
T_{m+1, n}=T_{m, n}-\frac{\left(T_{m, n}-T_{m, n+1}\right)^{2}}{T_{m, n}-2 T_{m, n+1}+T_{m, n+2}},
$$

then the sequences $T_{m, n}$ for fixed $m$ are integration rules of increasing order $O\left(h^{\widetilde{\beta}_{m}}\right)$. In case $\psi$ has logarithmic singularities $T_{m, n}$ is a rule of order $O\left(h^{3} /(\log h)^{2 m-5}\right)$ for $m \geq 2$.

Remarks. To eliminate the leading error term $c_{2} h^{2}$ of the trapezoidal approximation in the very first step there may be used the corresponding classical Romberg step.

Example 2. We use this method on the integral

$$
\int_{0}^{1} \frac{\exp x}{\sqrt{x}} d x=-\left.i \sqrt{\pi} \operatorname{Erf}(i x)\right|_{0} ^{1}=2.92530349181436 \ldots
$$


Erf denotes the error function. To use the trapezoidal rule we need a second primitive of $\psi(x)=1 / \sqrt{x}$, e.g. $4 x^{3 / 2} / 3$. We start with a step length $h=1 / 2$ and obtain

$\begin{array}{llll}T_{1, n} & T_{2, n} & T_{3, n} & T_{4, n} \\ 2.9811732544 & 2.9252857083 & 2.9253071463 & 2.9253034950 \\ 2.9395615282 & 2.9252965978 & 2.9253035659 & 2.9253034918 \\ 2.9289322995 & 2.9253019559 & 2.9253034964 & \\ 2.9262232288 & 2.9253031939 & 2.9253034921 & \\ 2.9255357475 & 2.9253034370 & & \\ 2.9253619756 & 2.9253034819 & & \\ 2.9253181878 & & & \\ 2.9253071791 & & & \end{array}$

\section{Implementation of the algorithm and error estimates}

A numerical realization of the formula $f * w_{\lambda}=R^{\#}\left(R f * W_{\lambda}\right)$ will now look like this.

Step 1: The convolution $R f * W_{\lambda}$ is numerically approximated with the method discussed in the previous Section. We write $R f^{*} \stackrel{h}{*} W_{\lambda}$ for the numerical result obtained with evaluation of $R f$ at points of distance $h$.

Step 2: For the backprojection $R^{\#}$ which requires an integration over $S^{1}$ a standard integration algorithm such as Romberg's method may be used. We write $R_{p}^{\#}$ for the numerical value of this integral with discretization step $\pi / p$.

Thus we have

$$
\left(f * w_{\lambda}\right)_{F B}:=R_{p}^{\#}\left(R f^{h} * W_{\lambda}\right)=w_{\lambda} * f+e_{1}+e_{2}
$$

with error terms

$$
\begin{aligned}
& e_{1}=R_{p}^{\#}\left(R f^{h} * W_{\lambda}-R f * W_{\lambda}\right) \\
& e_{2}=\left(R_{p}^{\#}-R^{\#}\right)\left(R f * W_{\lambda}\right) .
\end{aligned}
$$

We emphasize that $f * w_{\lambda}$ is exactly the value we want (the average of $f$ over the pixel) unlike as in the case of the standard filter (4) where $f * w_{b}$ is only an approximation of the value which is intended to be constructed (the value of $f$ in the center of the pixel). In the latter case there will always be additional artifacts resulting from the non-compactness of the support of the point-spread function $w_{b}$.

The error term $e_{1}$ is of the order of the method which we choose in the calculation of the convolution $\stackrel{h}{*}$. On the other hand the term $e_{2}$ is of the 
order of the method we use for the backprojection $R^{\#}$. Notice that $W_{\lambda} * R f$ (as a function of the angle $\theta$ ) is as smooth as $f$ is.

In order to accelerate the algorithm there is usually an interpolation step preceding the backprojection: For the angles $\theta_{i}=i \pi / p, i=1, \ldots p$, we first calculate the values $\alpha_{i k}:=W_{\lambda}{ }^{h} R f\left(\theta_{i}, s_{k}\right)$, where $s_{k}=k / q$ for $k=-q_{\max },-q_{\max }+1, \ldots,+q_{\max }$. Then we interpolate the values $s_{k}$ in the computation of the backprojection $R_{p}^{\#}$ :

$$
\left(f * w_{\lambda}\right)_{F B I}:=R_{p}^{\#} I_{h}\left(R f^{h} * W_{\lambda}\right)=w_{\lambda}+d_{1}+d_{2}+d_{3} .
$$

Here $I_{h}$ denotes e.g. the polynomial interpolation in the points $s_{k}$. The error terms are

$$
\begin{aligned}
d_{1} & =R_{p}^{\#} I_{h}\left(R f^{h} * W_{\lambda}-R f * W_{\lambda}\right) \\
d_{2} & =R_{p}^{\#}\left(I_{h}\left(R f * W_{\lambda}\right)-R f * W_{\lambda}\right) \\
d_{2} & =\left(R_{p}^{\#}-R^{\#}\right)\left(R f * W_{\lambda}\right) .
\end{aligned}
$$

$d_{3}$ is again error term $e_{2}$. The term $d_{1}$ is of the order of the method which we choose in the calculation of the convolution $*$. At last $d_{2}$ is of the order of the interpolation $I_{h}$. So in consideration of the sampling conditions (3) we should choose methods of the same order in the three numerical steps ${ }^{h}$, $I_{h}$ and $R_{p}^{\#}$.

Remark. Notice that the weights $\Delta_{h}^{2} \omega_{\lambda}\left(s_{i k}\right)\left(\omega_{\lambda}^{\prime \prime}=W_{\lambda}\right)$ which occur in the numerical calculation of $\alpha_{i k}:=W_{\lambda}{ }^{h} R f\left(\theta_{i}, s_{k}\right)$ may be calculated in advance and stored in an array since they are independent of the function $f$. Thus the algorithm is as fast as the classical backprojection method.

\section{Comparison of the methods}

It seems impossible to compare the two concepts of the classical filters and of the singular filters in terms of mathematical criterion. In the end it is the human eye which prefers one picture or another. In order to compare the quality of the different filters we can simulate a Radon transformation $R f$ of some function $f$. For example the Radon transform of the characteristic function of a circle $K$ of radius $r$ centered at the origin is given by

$$
R \chi_{K}(\theta, s)=2 \sqrt{r^{2}-\min \left(r^{2}, s^{2}\right)} .
$$

The function which we want to reconstruct by the different filters considered in the previous sections is the characteristic function of a set $A=\left(B_{1} \backslash B_{2}\right) \cup$ $\left(R \backslash B_{3}\right)$ where $R$ is a rectangle and the $B_{i}$ are balls such that $B_{1} \supset B_{2} \supset$ $R \supset B_{3}$. 
We have chosen quite large pixels to make visible the different philosophy of conventional filters on the one hand and singular filters on the other: With the conventional filter (4) a pixel is intended to have the gray-level of its center whereas the concept of the singular filters (11), (12), (13) guarantees that the gray-level of a pixel is the mean value over its area. The examples below also show that the numerical error at the same level of discretization is smaller for the singular filters than for the conventional filter (compare discussion Section 2).

The improvement in the quality of the pictures by the singular filters (11), (12), (13) in comparison to the conventional filter (4)-(5) is quite obvious (see Figures 1-4). (The conventional filter used in the reconstruction of the pictures below, is chosen according to the sampling conditions (2)-(3).)

Acknowledgment. I would like to thank Prof. C. Blatter for bringing computerized tomography to my attention and for many helpful discussions and valuable suggestions.

\section{References}

[1] Brown, R. L., Numerical optimization of the filter function used in the convolution filtered back projection algorithm for computed tomography, Thesis Univ. Wisconsin, Madison - Wisc. (1978).

[2] Faridani, A., Reconstructing from efficiently sampled data in parallel-beam computed tomography, Inverse Probl. Theoret. Imaging, Pitmans Research Notes in Math. Series 245 (1991), 68-102.

[3] Herman, G. T., Natterer, F. (eds.), Mathematical aspects of computerized tomography, Proceedings Oberwolfach, Lecture Notes in Medical Informatics 8 (1981), Berlin (etc.), Springer.

[4] Herman, G. T., Louis, A. K., Natterer, F. (eds.), Mathematical methods in tomography, Proceedings of a Conference Held in Oberwolfach, Lecture Notes in Math. 1497 (1991), Berlin (etc.), Springer.

[5] de Hoog, F., Weiss, R., Asymptotic expansions for product integration, Math. Comp. 27(122) (1973), 295-306.

[6] Hungerbühler, N., Numerical analysis of singular weighted integrals, Computing $\mathbf{5 3}$ (1994), 195-203.

[7] Madych, W. R., Summability and approximate reconstruction from Radon transform data, Contemp. Math. 113 (1990), 189-219

[8] Natterer, F., The Mathematics of Computerized Tomography, Teubner, Stuttgart, 1986.

[9] Smith, K. T., Reconstruction formulas in computed tomography, Proc. Sympos. Appl. Math. 27 (1982), 7-23.

[10] Smith, K. T., Keinert, F., Mathematical foundations of computed tomography, Appl. Optics 24 (1985), 3950-3957. 
NORBERT HUngerbüHLER

Mathematik Departement

ETH ZENTRUM

8092 ZÜRCH, SwitzerLAND

E-MAIL:BUHLER@MATH.ETHZ.CH 


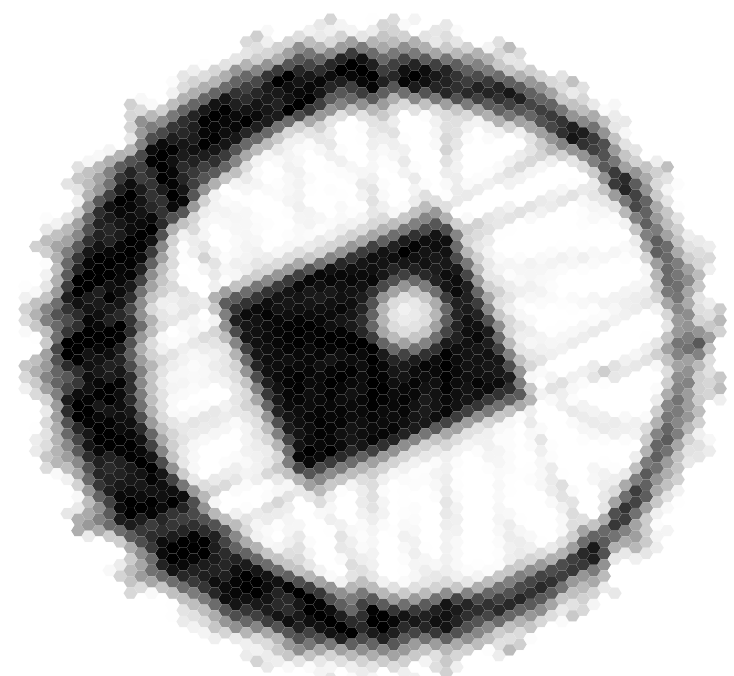

Figure 1. Image reconstruction with conventional filter (4). $R f$ sampled for 19 angles at 30 positions per angle.

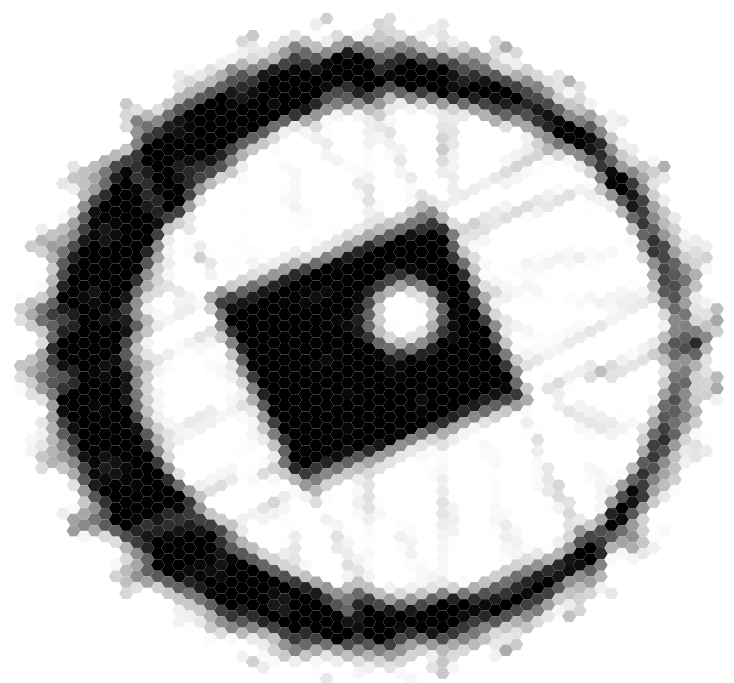

Figure 2. Image reconstruction with hexagonal filter (11). $R f$ sampled for 19 angles at 30 positions per angle. 


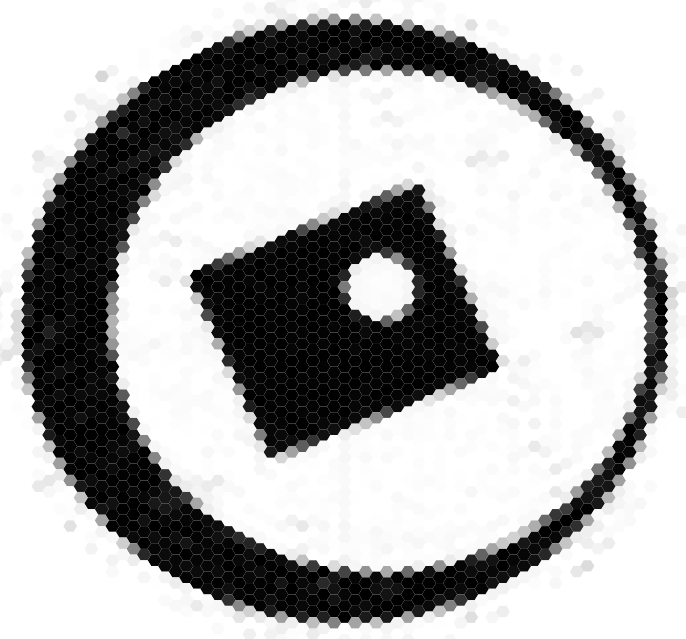

Figure 3. Image reconstruction with conventional filter (4). $R f$ sampled for 99 angles at 158 positions per angle.

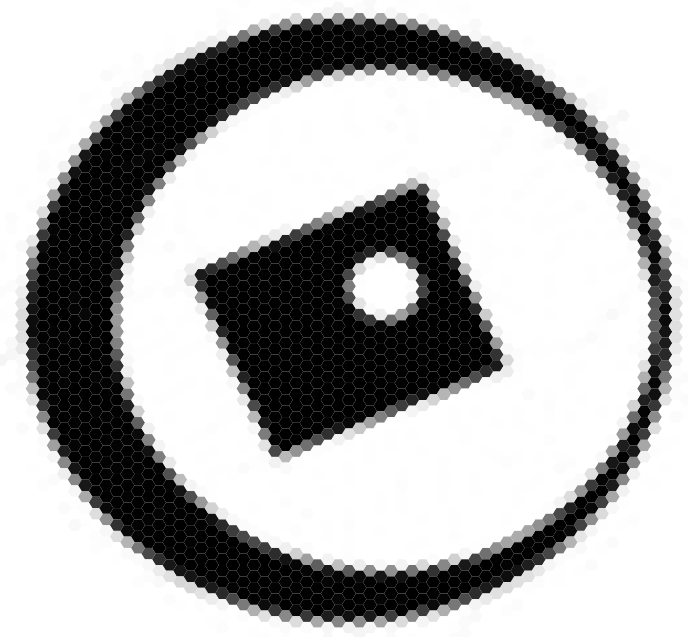

Figure 4. Image reconstruction with circular filter (13). $R f$ sampled for 99 angles at 158 positions per angle. 